\title{
ON THE NUMBER OF SOLUTIONS OF SOME INTEGRAL EQUATIONS ARISING IN RADIATIVE TRANSFER
}

\section{IOANNIS K. ARGYROS}

Department of Mathematics

New Mexico State University

Las Cruces, NM 88003

(Received on March 10, 1988 and in revised form on August 4, 1988)

ABSTRACT. We discuss the number of solutions of some nonlinear integral equations arising in the theories of radiative transfer, neutron transport and in the kinetic theory of gases.

KEYWORDS AND PHRASES. Radiative transfer, integral equation. A.M.S. 1980 CLASSIFICATION CODES: $65 \mathrm{R} 20,45 \mathrm{~L} 10$.

1. INTRODUCTION. In the theories of radiative transfer [1], [2] and neutron transport [3], [4] an important role is played by nonlinear integral equations of the form

$$
H(x)=1+x H(x) \int_{0}^{1} \frac{\psi(t) H(t)}{x+t} d t
$$

The known function $\psi$ is assumed to be nonegative, bounded, and measurable on $[0,1]$, and a positive, continuous solution $H$ of $(1.1)$ is sought.

Chandrasekhar's treatment of $(1.1)$ can be found in [2]. The first proof however of the existence of a solution of (1.1) was given by $M$. Crum, who considered the equation in the complex plane [5]. Crum also showed that if $\int_{0}^{1} \psi(t) d t<1 / 2$, then (1.1) has at most two solutions which are bounded in $[0,1]$ and in case $\int_{0}^{1} \psi(t) d t=1 / 2$, there is only one such solution. C. Fox [6] solved simpler equations in order to prove existence of solutions of (1.1). But the solution of Fox's equation are not necessarily solutions of (1.1)[1]. C. Stuart [7] gave a nonconstructive existence proof for (1.1) using the Leray-Schauder degree theory but did not discuss the number or location of solutions. B. Cahlon and M. Eskin [3] used a thenrem of Darbo for a set contraction map to prove a nonconstructive existence theorem for $(1.1)$.

Finally, C. Kelley [8] had solved some interesting generalizations of (1.1) using the solutions of finite rank approximations of solutions of (1.1). 
He re we consider the generalized equation:

$$
H(x)=1+x H(x) \int_{0}^{1} k(x, t) \psi(t) H(t) d t \text {. }
$$

The known kernal function $k(x, t)$ is a measurable function on $[0,1] x[0,1]$ satisfying (a) $0<k(x, t)<1$ for al1 $x, t \in[0,1]$,

and

(b) $k(x, t)+k(t, x)=1$ for all $x, t \in[0,1]$.

We show that whenever $\int_{0}^{1} \psi(t) d t<1 / 2$, a minimal solution $H$ can be found using a specific iteration.

Finally, under the same assumption, we provide a way of constructing new nonminimal solutions $H$ of (1.1) in terms of the minimal solution.

\section{BASIC RESULTS.}

We denote by $C[0,1]$ the Banach space of all real continuous functions on $[0,1]$ with the maximum norm

$$
u=\max _{0<t<1}|u(t)| \text {. }
$$

We now list the following well-known theorem whose proof can be found in [2, pp. 106-107].

THEOREM 1. If $\mathrm{H}$ is a solution of $(1.2)$, then either

$$
\int_{0}^{1} \psi(t) H(t) d t=1-\left[1-2 \int_{0}^{1} \psi(t) d t\right]^{1 / 2}
$$

or

$$
\int_{0}^{1} \psi(t) H(t) d t=1+\left[1-2 \int_{0}^{1} \psi(t) d t\right]^{1 / 2} .
$$

A necessary condition that (1.2) has a solution is that

$$
\int_{0}^{1} \psi(t) d t<1 / 2 \text {. }
$$

A function $H \in C[0,1]$ satisfies the equation

$$
H(x)^{-1}=\left[1-2 \int_{0}^{1} \psi(t) d t\right]^{1 / 2}+\int_{0}^{1} k(t, x) \psi(t) H(t) d t
$$

if and only if $\mathrm{H}$ satisfies (1.2) and (2.1).

Chandrasekhar in [2], after proving that a solution H of (1.1) satisfies either (2.1) or (2.2), claims that, in fact, H must satisfy (2.1). This claim is not true because as we show, there always exists a solution $H$ satisfying (2.1), but in many cases there exists a second solution $\tilde{H}$ satisfying (2.2) and not (2.1).

Let $<$ be the natural partial ordering on $c[0,1]$, that is, if

$p_{1}, p_{2} \in C[0,1]$, then $p_{1}<p_{2}$ if $p_{1}(x)<p_{2}(x)$ for all $x \in[0,1]$ and define the following:

$$
\begin{gathered}
d=\left[1-2 \int_{0}^{1} \psi(t) d t\right]^{1 / 2}, \\
D=\{p \in C[0,1] / p(x)>d, x \in[0,1]\},
\end{gathered}
$$

the operator

$$
\begin{gathered}
R: D \rightarrow c[0,1] \text { by } \\
R(p(x))=1+p(x) \int_{0}^{1} k(x, t) \psi(t) p(t) d t, p \in D
\end{gathered}
$$

and for $d>0$, define the operator $F: D \rightarrow C[0,1]$ by 


$$
F(p(x))=d+\int_{0}^{1} k(t, x) \psi(t)(p(t))^{-1} d t, p \in D
$$

It is routine to verify that $R$ is isotone, that is if $p_{1}<p_{2}$ then $R\left(p_{1}\right)<R\left(p_{2}\right)$ and $F$ is antitone, that is if $p_{1} \leqslant p_{2}$ then $F\left(p_{2}\right)<F\left(p_{1}\right)$.

Finally, denote by 1 (respectively, d) the function with constant value 1 (respectively, d).

We can now prove the proposition:

PROPOSITION. Assume that the kernel function $k(x, t)$ is as in the introduction and satisfies the condition

$$
|k(x, t)-k(y, t)|<b|x-y| \text { for all } x, y, t \in[0,1]
$$

and some $b>0$. Then the sequence $R^{n}(1), n=1,2, \ldots$ is equicontinuous.

PROOF. Let $H$ be a solution satisfying (1.2) and (2.1) and $A=\{p \in C[0,1] / 1<p<H\}$.

Define $Q: A \rightarrow C[0,1]$ by

$$
Q(p(x))=\int_{0}^{1} k(x, t) \psi(t) p(t) d t, x \in[0,1], p \in A .
$$

Let $\varepsilon>0$, then there exists $a, 0<a<1$, such that

$\int_{0}^{a} \psi(t) H(t) d t<\frac{\varepsilon}{4}$ and $\int_{a}^{1} \psi(t) A(t) d t>0$. Then for $x, y \in[0,1]$,

$$
\begin{aligned}
& |Q(p(x))-Q(p(y))|=\left|\int_{0}^{1}(k(x, t)-k(y, t)) \psi(t) p(t) d t\right| \\
& \quad<\int_{0}^{a}(|k(x, t)|+|k(y, t)|) \psi(t) H(t) d t+\int_{a}^{1}|k(x, t)-k(y, t)| \psi(t) H(t) d t \\
& \quad<2 \int_{0}^{a} \psi(t) H(t) d t+b \int_{a}^{1}|x-y| \psi(t) \psi(t) H(t) d t \\
& \quad<\frac{\varepsilon}{2}+\frac{\varepsilon}{2}=\varepsilon,
\end{aligned}
$$

by choosing, $|x-y|<\delta=\frac{\varepsilon}{2 b}\left(\int_{a}^{1} \psi(t) H(t) d t\right)^{-1}$. That is the set $Q(A)$ is equicontinuous.

$$
\begin{aligned}
& \text { Let } p \in A \text { and } x \in[0,1], \text { then } \\
& \qquad \begin{aligned}
& Q(p(x))=\int_{0}^{1} k(x, t) \psi(t) p(t) d t<\int_{0}^{1} k(x, t) \psi(t) H(t) d t \\
&<\int_{0}^{1} \psi(t) H(t) d t=1-d<1 .
\end{aligned}
\end{aligned}
$$

Therefore there exists $c, 0<c<1$, such that $Q(p(x))<c$ for all $p \in A$ and $x \in[0,1]$.

For any $\varepsilon_{0}>0$, there exists $\delta_{0}>0$ such that for every $g \in Q(A)$, $|g(x)-g(y)|<|| H \|^{-1}(1-c) \varepsilon_{0}$ if $|x-y|<\delta_{0}$ (since $Q(A)$ is equicontinuous). The function $R(1)$ is continuous and hence uniformly continuous, therefore there exists $0<\delta_{1}<\delta_{0}$ such that

$$
|R(1(x))-R(1(y))|<\varepsilon_{0} \text { if }|x-y|<\delta_{1}
$$


We shall show that the same $\delta_{1}$ works for $\varepsilon_{0}$ and $R^{n+1}(1)$ if

$$
\left|s^{k}(1(x))-s^{k}(1(y))\right|<\varepsilon_{0} \text { if }|x-y|<\delta_{1} \text { for } k=1,2, \ldots n \text {. }
$$

Set $p=R^{n}(1)$. Then if $|x-y|<\delta_{1}$,

$|R(p(x))-R(p(y))|=|p(x) Q(p(x))-p(y) Q(p(y))|$

that is,

$$
\begin{aligned}
& <|p(x) Q(p(x))-p(x) Q(p(y))|+\mid p(x) Q(p(y))-p(y) Q(p(y))) \\
& <p(x)|Q(p(x))-Q(p(y))|+Q(p(y))|p(x)-p(y)| \\
& <\|H\|\|H\|^{-1}(1-c) \varepsilon_{0}+c \varepsilon_{0}=\varepsilon_{0},
\end{aligned}
$$

$$
\left|R^{n+1}(1(x))-R^{n+1}(1(y))\right|<\varepsilon_{0} \text { if }|x-y|<\delta_{1}
$$

which completes the induction and the proof of the proposition.

THEOREM. 2. As sume that the kernel function $k(x, t)$ is as in the proposition. Then the following are true:

(a) equation (1.2) has exactly one solution $H$ satisfying (2.1) if and only if (2.3) holds. Moreover, the increasing sequence $R^{n}(1), n=0,1,2, \ldots$ converges to $\mathrm{H}$; and

(b) if inequality holds in $(2.3)$, the sequence $\mathrm{F}^{\mathrm{n}}(\mathrm{d}), \mathrm{n}=0,1,2, \ldots$ converges to $\mathrm{H}^{-1}$ and

$$
\left|H^{-1}(x)-F^{n}(d(x))\right|<\left|F^{n}(d(x))-F^{n+1}(d(x))\right|, x \in[0,1] .
$$

PROOF. (A). If (1.2) has a solution $H$, then by Theorem $1, \int_{0}^{1} \psi(t) d t<1 / 2$.

CASE 1. Assume $\int_{0}^{1} \psi(t) d t<1 / 2$. It can eas $11 y$ be verified that since $F 1 s$ antitone:

$$
d<F^{2}(d)<F^{4}(d)<F^{6}(d)<\ldots<F^{7}(d)<F^{5}(d)<F^{3}(d)<F(d) .
$$

Working as in the proposition we can easily show that the bounded set

$$
N=\{F(p) / d<p<F(d)\}
$$

is equicontinuous. Then the sequences $F^{2 n}(d), n=1,2, \ldots$

and $F^{2 n+1}(d), n=0,1,2, \ldots$ have convergent subsequences converging to the functions $v$ and $w$ respectively. From the monotonicity of the above sequences and the continuity of $F$ we obtain

$$
\begin{gathered}
F^{2 n}(d) \rightarrow v, \\
F^{2 n+1}(d) \rightarrow w, \\
d<v<w, \\
F(v)=w
\end{gathered}
$$

and

$$
F(w)=v .
$$

The function $v$ has minimum value greater than zero, so that there exists a largest number $q, 0<q<1$, with $q w \leqslant v$. If $q=1$, then $w<v<w$, that is $v=w$. 
If $q<1$, define on the domain of $F$ the operator $F_{1}$ by

Then

$$
F_{1}(p)=F(p)-d
$$

$$
\begin{aligned}
v=d & +F_{1}(w)>d+F_{1}\left(q^{-1} v\right)=d+q F_{1}(v) \\
& =(1-q) d+q\left(d+F_{1}(v)\right)=(1-q) d+q w \\
& <e w+q w=(e+q) w
\end{aligned}
$$

for some $e>0$. But this contradicts the maximality of $q$. Therefore,

$$
\begin{aligned}
\mathrm{F}(\mathrm{v}) & =\mathrm{v}=\mathrm{w}, \\
\mathrm{H} & \equiv \mathrm{v}^{-1}
\end{aligned}
$$

is a solution of (1.2), satisfying (2.1), and the sequence $F^{n}(d), n=0,1,2, \ldots$ converges to $\mathrm{H}^{-1}$. Inequality $(2.5)$ follows from the fact that $\mathrm{F}^{2 \mathrm{k}}(\mathrm{d})<\mathrm{H}^{-1} \leqslant \mathrm{~F}^{2 \mathrm{k}+1}(\mathrm{~d})$, for $\mathrm{k}=1,2,3, \ldots$.

CASE 2. As sume that $\int_{0}^{1} \psi(t) d t=1 / 2$. Let $\left\{c_{n}\right\}, n=1,2, \ldots$ be a strictly increasing sequence of positive numbers converging to 1 , and consider the functions $c_{n} \psi, n=1,2,3, \ldots$.

Since $\int_{0}^{1} c_{n} \psi(t) d t=1 / 2 c_{n}<1 / 2$, it follows from Case 1 that the equation

$$
H(x)=1+H(x) \int_{0}^{1} k(x, t) c_{n} \psi(t) H(t) d t
$$

has a solution $H_{n}$ for $n=1,2,3, \ldots$. Then for each $x \in[0,1] h_{n}(x)>1$ and

$$
\begin{aligned}
\left(H_{n}(x)\right)^{-1} & =\left[1-2 \int_{0}^{1} c_{n} \psi(t) d t\right]^{1 / 2}+\int_{0}^{1} k(t, x) c_{n} \psi(t) H_{n}(t) d t \\
& >c_{n} \int_{0}^{1} k(t, x) \psi(t) d t>c_{1} \int_{0}^{1} k(t, x) \psi(t) d t .
\end{aligned}
$$

Therefore, there exists $r>0$ such that $\left(H_{n}(x)\right)^{-1}>r$ for each $x \in[0,1]$ and each $\mathrm{n}=1,2,3, \ldots$.

Set $M=\{p \in C[0,1) / r<p(x) \leqslant 1, x \in[0,1]\}$. Then $H_{n}^{-1} \in M, n=1,2, \ldots$. Define $F: M \rightarrow C[0,1]$ by

$$
F(p(x))=\int_{0}^{1} k(t, x) \Psi(t)(p(t))^{-1} d t, p \in M .
$$

It is easy to verify that the set $F(M)$ is bounded and equicontinuous.

Also, for each $n$,

$$
\begin{aligned}
H_{n}^{-1}(x)= & {\left[1-2 \int_{0}^{1} c_{n} \psi(t) d t\right]^{1 / 2}+\int_{0}^{1} k(t, x) c_{n} \psi(t) H_{n}(t) d t } \\
& =\left[1-2 \int_{0}^{1} c_{n} \psi(t) d t\right]^{1 / 2}+c_{n} F\left(H_{n}^{-1}\right)(x) .
\end{aligned}
$$

Si nce $F\left(H_{n}^{-1}\right) \in F(M)$ for each $n$, some subsequence $F\left(H_{n_{j}}^{-1}\right), j=1,2, \ldots$, of $F\left(H_{n}^{-1}\right)$, $n=1,2, \ldots$ converges in $C[0,1]$ to some point $\mathrm{H}_{0}^{-1}$, so that $\mathrm{H}_{\mathrm{j}}^{-1} \rightarrow \mathrm{H}_{0}^{-1}$. Then the sequence $F\left(H_{n_{j}}^{-1}\right), j=1,2, \ldots$ converges to $F\left(H_{0}^{-1}\right)$ and $H_{0}^{-1}$; that is, 


$$
\mathbf{F}\left(\mathrm{H}_{0}^{-1}\right)=\mathrm{H}_{0}^{-1}
$$

Then $H_{0}$ satisfies $(1.2),(2.1)$ and $(2.2)$.

Therefore there exists a positive function $H$ satisfying (1.2) and (2.1) whenever $\psi$ satisfies $(2.3)$.

(B). As sume (2.3) holds, and suppose $H$ satisfies (1.2) and (2.1). Since $1 \leqslant \mathrm{H}$ and $1<\mathrm{R}(1)$, it follows from the fact that $\mathrm{R}$ is isotone that

$$
1<\mathrm{R}(1)<\mathrm{R}^{2}(1)<\mathrm{R}^{3}(1)<\ldots<\mathrm{H} \text {. }
$$

Since the sequences $R^{n}(1), n=1,2, \ldots$ is uniformly bounded and equicontinuous there is a convergent subsequence, say $R^{n_{k}} \rightarrow h<H$, and, since the sequence $R^{n}(1), n=0,1$, 2 , ... is nondecreasing, the entire sequence converges to $h$. It follows from the continuity of $R$ that $R(h)=h$. Now $h$ must satisfy either (2.1) or (2.2), and since $0<h<H, h$ must satisfy $(2.1)$. Therefore, for $x \in[0,1]$,

$$
\begin{aligned}
h^{-1}(x) & =\left[1-2 \int_{0}^{1} \psi(t) d t\right]^{1 / 2}+\int_{0}^{1} k(t, x) \psi(t) h(t) d t \\
< & {\left[1-2 \int_{0}^{1} \psi(t) d t\right]^{1 / 2}+\int_{0}^{1} k(t, x) \psi(t) H(t) d t=H^{-1}(x), }
\end{aligned}
$$

that is, $h^{-1}<H^{-1}$. Together with the inequality $h<H$, this implies $h=H$.

We have proved that $H$ is the only function satisfying both (1.2) and (2.1), and that the increasing sequence $\mathrm{R}^{\mathrm{n}}(1), \mathrm{n}=0,1,2, \ldots$ converges to $\mathrm{H}$ which completes the proof of the theorem.

COROLLARY. Suppose that $\psi_{1}$ and $\psi_{2}$ are nonnegative, bounded, measurable functions on $[0,1]$ such that $\psi_{1}(t)<\psi_{2}(t)$ almost everywhere in $[0,1]$ and such that $\int_{0}^{1} \psi(t) d t<1 / 2, i=1,2$. Let $H_{i}$ be the unique solution of equations (1.2) and (2.1) corresponding to $\psi=\psi_{1}, 1=1,2$.

Then,

$$
\mathrm{H}_{1}<\mathrm{H}_{2} \bullet
$$

PROOF. Define $R_{1}: C[0,1] \rightarrow c[0,1], 1=1,2$, by

$$
R_{i}(p(x))=1+p(x) \int_{0}^{1} k(x, t) \Psi_{1}(t) p(t) d t, p \in c[0,1]
$$

If $p_{1}$ and $p_{2}$ are nonnegative functions in $c[0,1]$ with $p_{1}<p_{2}$, then

$R_{1}\left(p_{1}\right)<R_{2}\left(p_{2}\right)$. Hence $R_{1}(1)<R_{2}(1), R_{1}^{2}(1)<R_{2}^{2}(1)$, and in general,

$R_{1}^{n}(1)<R_{2}^{n}(1)$. Since the increasing sequence $R_{1}^{n}(1)$, converges to $H_{1,1}=1$, 2 , it follows that $\mathrm{H}_{1}<\mathrm{H}_{2}$.

Note that if $\int_{0}^{l} \psi(t) d t=1 / 2$, it follows from the previous results that the function $H$ satisfying (1.2) and (2.1) is the unique solution of (1.2), since, in this case (2.1) and (2.2) reduce to the same equation. However, if $\int_{0}^{1} \psi(t) d t<1 / 2$, equation $(1.2)$ may have two distinct solutions.

THEOREM 3. As sume:

(a) $\int_{0}^{1} \Psi(t) d t<1 / 2$ and $H$ is the unique solution of (1.2) and (2.1); 
(b) the following estimate is true

$$
\int_{0}^{1} \frac{\psi(t)}{1-t} H(t) d t>1
$$

and

(c) there exists functions $\varphi_{1}, \varphi_{2}, \varphi_{3} \in \mathrm{C}[0,1]$ such that

$k(x, t)\left[\varphi_{2}(x)(1-k t)-\left(1+\varphi_{1}(t)\right)\right]+\varphi_{3}(x)=0$, for all $x, t \in[0,1]$

$$
\varphi_{1}(\mathrm{x})+\mathrm{kx}>0, \text { for a11 } \mathrm{x} \in(0,1], \varphi_{1}(0)=0
$$

and

$$
\begin{array}{r}
\left(1+\varphi_{1}(x)\right)\left[\varphi_{2}(x)(H(x)-1)+\varphi_{3}(x) H(x)\right]= \\
\left(H_{1}(x)-1\right)(1-k x) \text { for all } x \in[0,1]
\end{array}
$$

where $k$ is the unique number in $(0,1)$ for which

$$
\int_{0}^{1} \frac{\psi(t)}{1-k t} H(t) d t=1
$$

and the function $\mathrm{H}_{1}$ is given by

$$
H_{1}(x)=\frac{1+\varphi_{1}(x)}{1-k x} H(x), x \in[0,1] \text {. }
$$

Then $H_{1}$ is a solution of (1.2) and (2.2) and

$$
\mathrm{H}_{1}(\mathrm{x})>\mathrm{H}(\mathrm{x}), \mathrm{x} \in(0,1], \mathrm{H}_{1}(0)=\mathrm{H}(0) \text {. }
$$

PROOF. By the monotone convergence theorem

$$
\lim _{k \rightarrow 1} \int_{0}^{1} \frac{\psi(t)}{1-k t} H(t) d t=\int_{0}^{1} \frac{\psi(t)}{1-t} H(t) d t
$$

since $(1-k t)^{-1}$ increases monotonically with $k, 0<k<1$, If $(2.6)$ holds, since

$$
\int_{0}^{1} \frac{\psi(t)}{1-0 \cdot t} H(t) d t=1-\left[1-2 \int_{0}^{1} \psi(t) d t\right]^{1 / 2}<1,
$$

and since the function $f:(0,1) \rightarrow \mathbb{R}$ defined by

$$
f(k)=\int_{0}^{1} \frac{\psi(t)}{1-k t} H(t) d t
$$

is strictly increasing, there exists a unique $k \in(0,1)$, for which (2.10) holds. Let $H_{1}$ be defined as in (2.11). Applying a trick used in [5], [9], we find that for each $x \in[0,1]$

$$
\int_{0}^{1} k(x, t) \psi(t) H_{1}(t) d t=\varphi_{2}(x) \int_{0}^{1} k(x, t) \psi(t) H(t) d t+\varphi_{3}(x) \int_{0}^{1} \frac{H(t)}{1-k t} \psi(t) d t
$$




$$
\begin{gathered}
=\varphi_{2}(x) \int_{0}^{1} k(x, t) \psi(t) H(t) d t+\varphi_{3}(x) \\
=\varphi_{2}(x)\left[1-\frac{1}{H(x)}\right]+\varphi_{3}(x)=1-\frac{1}{H_{1}(x)},
\end{gathered}
$$

(by (2.7) and (2.9)) that is, $H_{1}$ satisfies (1.2). Since $H_{1}$ must satisfy either (2.1) or (2.2) and since $H_{1}(x)>H(x), x \in[0,1]$ (by $(2.8)$ ), $H_{1}$ satisfies (2.2) and the proof of the theorem is completed.

REMARK. By choosing the kernel function $k(x, t)$ to be

$$
k(x, t)=\frac{x}{x+t}, x, t \in[0,1]
$$

we observe that the conditions (a) and (b) in the introduction are satisfied and that the equation $(1.2)$ reduces to equation $(1.1)$.

Moreover the conditions $(2.7),(2.8)$, and $(2.9)$ can then be satisfled if we choose

$$
\begin{gathered}
\varphi_{1}(x)=k x \\
\varphi_{2}(x)=\frac{1-k x}{1+k x}
\end{gathered}
$$

and

$$
\varphi_{3}(x)=\frac{2 k x}{1+k x}
$$

\section{REFERENCES}

1. BUSBRIDGE, L.W. The Mathematics of Radiative Transfer. Cambridge Pub1. Cambridge, England, 1960.

2. CHANDRASEKHAR, S. Radiative Transfer, Oxford Univ. Press, London, 1950.

3. CAHLON, B. AND ESKIN, M. Existence Theorems for an Integral Equation of the Chandrasekhar Hequation with Perturbation. J. Math. Anal. and Applic. 83(1981), 159-171.

4. ARGYROS, I.K. Quadratic Equations and Applications to Chandrasekhar's and Related Equations. Bul1. Austra 1. math. Sco., Vo1. 32, 2(1985), 275-292.

5. CRUM, M. On an Integral Equation of Chandrasekhar. Quart. J. Math., 18(1947), 244-252.

6. FOX, C. A Solution of Chandrasekhar's Integral Equation. Trans. Amer. Math. Soc., 99(1961), 285-291.

7. STUART, C.A. Existence Theorems for a Class of Nonlinear Integral Equations. Math. Z. 137(1974), 49-66.

8. KELLEY, C.T. Approximation of Solutions of Some Quadratic Integral Equations in Transport Theory. J. Integr. Eq.2 4(1982), 221-237.

9. LEGGETT, R.W. A New Approach to the Hequation of Chandrasekhar. S.I.A.M. J. Math., 7, 4(1976), 542-550.

10. CASE, K.M. \& ZWEIFEL, P.F. Linear Transport Theory, Addison-Wesley Publ., MA, 1967.

11. KANTOROVICH, L.V. AND AKILOV, G.P. Functional Analysis in Normed Spaces. Pergamon Press, London, 1964.

12. KURATOWSKI, C. Sur les Espaces Complets. Fund. Math., 15(1930), 301-309. 


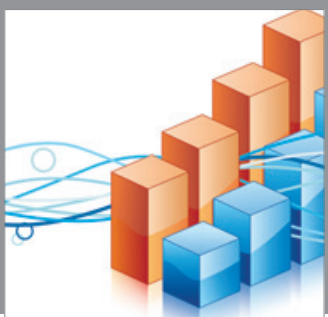

Advances in

Operations Research

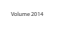

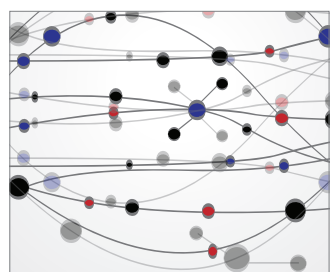

\section{The Scientific} World Journal
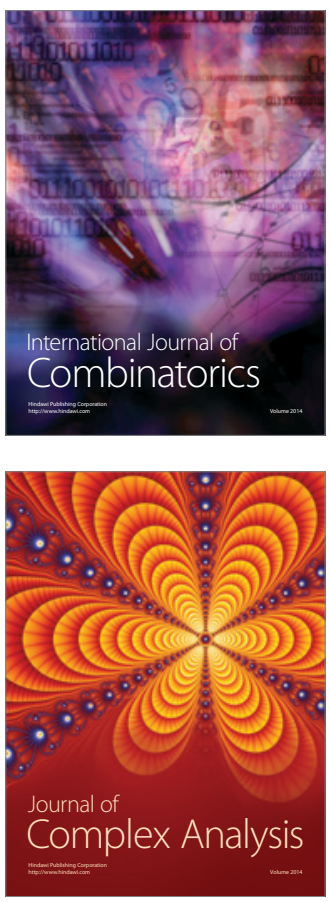

International Journal of

Mathematics and

Mathematical

Sciences
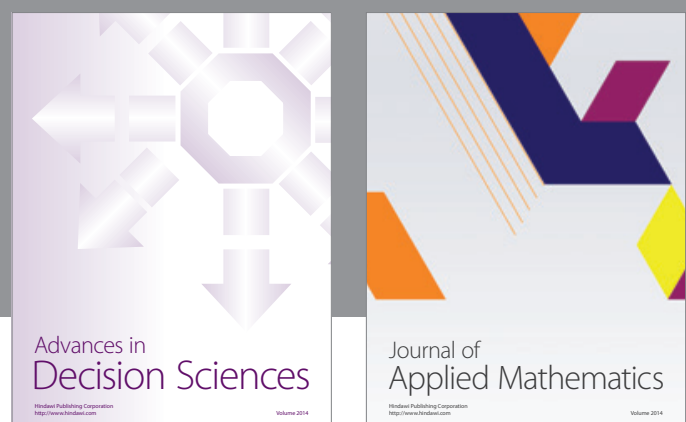

Journal of

Applied Mathematics
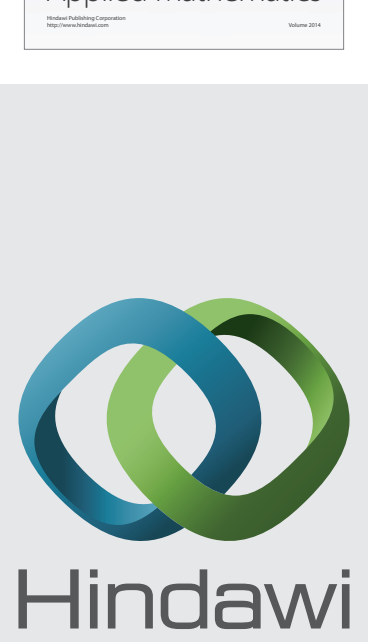

Submit your manuscripts at http://www.hindawi.com
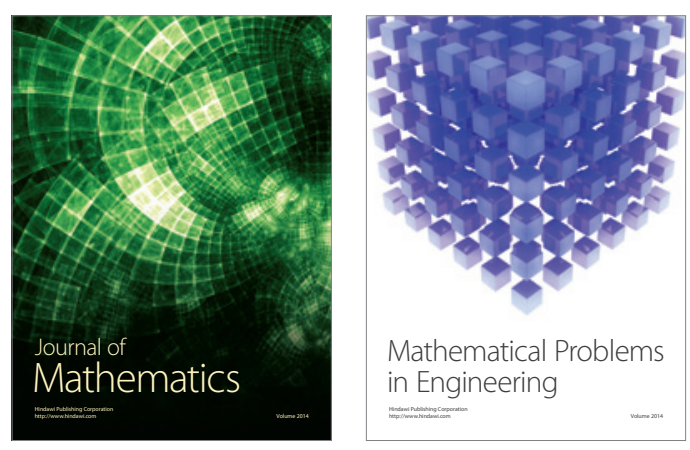

Mathematical Problems in Engineering
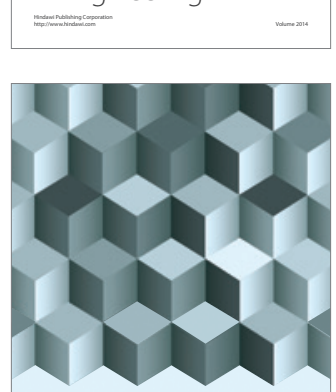

Journal of

Function Spaces
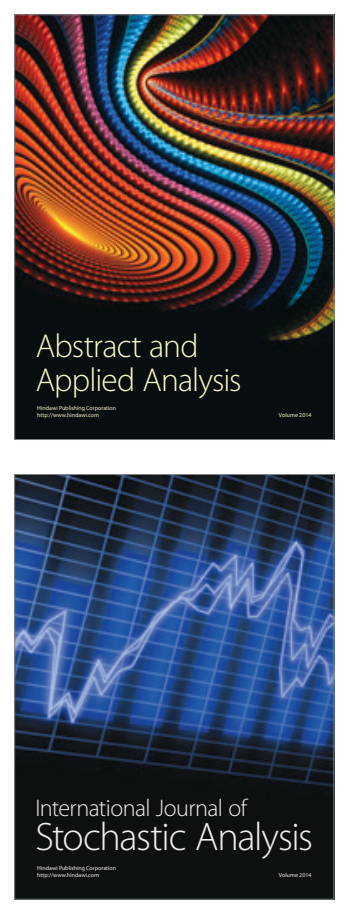

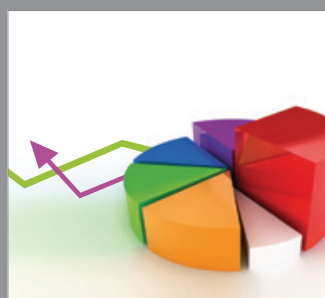

ournal of

Probability and Statistics

Promensencen
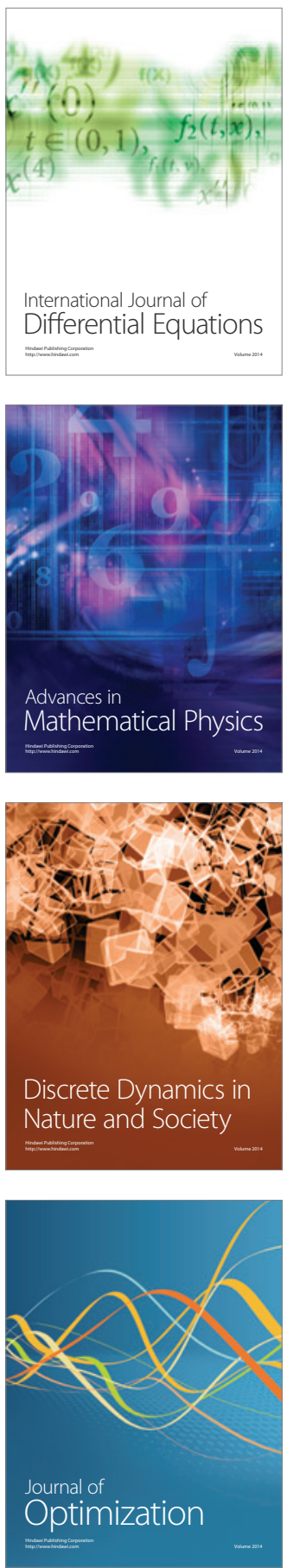